\title{
Solar abundances of rock-forming elements, extreme oxygen and hydrogen in a young polluted white dwarf
}

\author{
J. Farihi, ${ }^{\star}{ }^{\star} \dagger$ D. Koester, ${ }^{2}$ B. Zuckerman, ${ }^{3}$ L. Vican, ${ }^{3}$ B. T. Gänsicke, ${ }^{4}$ N. Smith, ${ }^{5}$ \\ G. Walth ${ }^{5}$ and E. Breedt ${ }^{4}$ \\ ${ }^{1}$ Department of Physics and Astronomy, University College London, London WC1E 6BT, UK \\ ${ }^{2}$ Institut für Theoretische Physik und Astrophysik, University of Kiel, D-24098 Kiel, Germany \\ ${ }^{3}$ Department of Physics and Astronomy, University of California, Los Angeles, CA 90095, USA \\ ${ }^{4}$ Department of Physics, University of Warwick, Coventry CV4 7AL, UK \\ ${ }^{5}$ Steward Observatory, University of Arizona, Tucson, AZ 85721, USA
}

Accepted 2016 August 26. Received 2016 August 26; in original form 2016 May 29

\begin{abstract}
The $T_{\text {eff }}=20800 \mathrm{~K}$ white dwarf WD $1536+520$ is shown to have broadly solar abundances of the major rock-forming elements $\mathrm{O}, \mathrm{Mg}, \mathrm{Al}, \mathrm{Si}, \mathrm{Ca}$, and $\mathrm{Fe}$, together with a strong relative depletion in the volatile elements $\mathrm{C}$ and $\mathrm{S}$. In addition to the highest metal abundances observed to date, including $\log (\mathrm{O} / \mathrm{He})=-3.4$, the helium-dominated atmosphere has an exceptional hydrogen abundance at $\log (\mathrm{H} / \mathrm{He})=-1.7$. Within the uncertainties, the metal-to-metal ratios are consistent with the accretion of an $\mathrm{H}_{2} \mathrm{O}$-rich and rocky parent body, an interpretation supported by the anomalously high trace hydrogen. The mixed atmosphere yields unusually short diffusion time-scales for a helium atmosphere white dwarf, of no more than a few hundred years, and equivalent to those in a much cooler, hydrogen-rich star. The overall heavy element abundances of the disrupted parent body deviate modestly from a bulk Earth pattern, and suggest the deposition of some core-like material. The total inferred accretion rate is $4.2 \times 10^{9} \mathrm{~g} \mathrm{~s}^{-1}$, and at least four times higher than for any white dwarf with a comparable diffusion time-scale. Notably, when accretion is exhausted in this system, both metals and hydrogen will become undetectable within roughly $300 \mathrm{Myr}$, thus supporting a scenario where the trace hydrogen is related to the ongoing accretion of planetary debris.
\end{abstract}

Key words: stars: abundances - circumstellar matter-stars: individual: (WD 1536+520) planetary systems - white dwarfs.

\section{INTRODUCTION}

A decade of observational and theoretical studies by many astronomers has shown that, over a wide range of effective stellar temperatures, the presence of heavy elements in white dwarf atmospheres is evidence for orbiting planetary systems (Jura \& Young 2014; Vanderburg et al. 2015; Veras et al. 2015; Farihi 2016). With this relatively recent shift in paradigm, the discovery of the prototype, metal-lined white dwarf by van Maanen (1917) nearly a century ago - while not a planet detection itself, but the signature of accreted planetary debris - is arguably the first astronomical evidence of the presence of planetary systems around other stars (Zuckerman 2015).

^E-mail: j.farihi@ucl.ac.uk

$\dagger$ STFC Ernest Rutherford Fellow.
According to all dynamical models that deliver sufficient planetesimal masses into the innermost system, where it can be accreted, each exoplanetary system hosted by a metal-enriched white dwarf must harbour at least a belt of minor bodies and one major planet (Bonsor, Mustill \& Wyatt 2011; Debes, Walsh \& Stark 2012; Veras et al. 2013; Frewen \& Hansen 2014). The gravitational field of the planet(s) can perturb the orbits of the planetesimals on to orbits passing near the white dwarf so that they are tidally disrupted. Spitzer and complementary ground-based observations have established a firm connection between the atmospheric heavy elements in white dwarfs and the presence of dust and gas within the tidal radius of the star (Gänsicke et al. 2006; Jura, Farihi \& Zuckerman 2007; von Hippel et al. 2007; Farihi, Jura \& Zuckerman 2009).

Because the metal-to-metal sinking time-scales vary by no more than a factor of a few, the relative, steady-state abundances of the accreted planetary debris can be analytically linked to those observed in the polluted atmosphere (Koester 2009), thus making the stellar surface an effective mirror of planetesimal composition. The 
first detailed abundance study of any metal-enriched white dwarf was carried out for the current record holder for number (16) of detected heavy elements, GD 362, demonstrating that the debris was broadly terrestrial-like (Zuckerman et al. 2007). Since then, the broad pattern of bulk, Earth-like compositions has been seen - especially with ultraviolet Hubble Space Telescope observations - in several more stars with five or more heavy elements $(\mathrm{O}, \mathrm{Mg}, \mathrm{Si}, \mathrm{Ca}$, and $\mathrm{Fe}$ ) that indicate melting and differentiation among extrasolar, rocky planetesimals, and a diversity of overall compositions similar to different classes of solar system meteorites (Gänsicke et al. 2012; $\mathrm{Xu}$ et al. 2013).

Importantly, while most polluted white dwarfs appear to be contaminated by debris from parent bodies that were relatively poor in $\mathrm{H}_{2} \mathrm{O}$ and other volatiles (Jura \& Xu 2012), there is at least one case where substantial $\mathrm{H}_{2} \mathrm{O}$ can be confirmed in an otherwise volatile- and carbon-poor planetesimal. The debris orbiting and polluting the atmosphere of GD 61 originated in a rocky minor planet, roughly the size of Vesta and containing approximately 26 per cent water by mass (Farihi, Gänsicke \& Koester 2013a). Another polluted white dwarf with a substantial oxygen excess is SDSS J124231.07+522626.6, where the parent body likely had an even higher water content (Raddi et al. 2015). Such water-rich asteroids are important as potential building blocks of habitable planetary surfaces, especially if most small and rocky planets form dry as did the Earth (Morbidelli et al. 2000).

This paper reports the identification and analysis of $\mathrm{H}, \mathrm{O}, \mathrm{Mg}$, $\mathrm{Al}, \mathrm{Si}, \mathrm{Ca}, \mathrm{Ti}, \mathrm{Cr}$, and $\mathrm{Fe}$ in the helium atmosphere white dwarf WD $1536+520$. These elements are found to be accreting at a rate higher than any yet measured in a white dwarf with relatively short sinking time-scales, and producing atmospheric metal abundances comparable to those of the Sun. The data are consistent with a refractory-rich parent body with a modest fraction of $\mathrm{H}_{2} \mathrm{O}$. Section 2 presents spectroscopic observations from several facilities that resulted in the detection of all the major rock-forming elements, and strong upper limits on key volatiles. The atmospheric modelling is discussed in Section 3, along with the determination of stellar parameters, and elemental abundances within the star and the disrupted parent body. The paper explores the so-far unique properties of this star as something of a transition object between helium- and hydrogen-rich polluted white dwarfs, with the conclusions presented in Section 4.

\section{OBSERVATIONS}

WD 1536+520 was first identified in the Second Byurakan Sky Survey (SBS 1536+520; Balayan 1997; Stepanian et al. 1999) in 1992 and correctly typed as a DBA (strongest lines He I, weaker lines of $\mathrm{H}$ ) white dwarf from a low-resolution, $R \approx 400$ spectrum. It was spectroscopically observed as part of the Sloan Digital Sky Survey in 2002 (SDSS 153725.71+515126.9; Eisenstein et al. 2006), and exhibits lines of $\mathrm{Mg}, \mathrm{Si}$, and $\mathrm{Ca}$ in these $R \approx 2000$ data (Gänsicke et al., in preparation), yielding a full spectral type of DBAZ. Given that the SDSS ugriz photometry alone results in a temperature estimate of $22000 \mathrm{~K}$ (Girven et al. 2011), the presence of these metal-absorption features in a modest resolution spectrum is remarkable - at similar $T_{\text {eff }}$ and irrespective of atmospheric composition, the detection of atmospheric metals in white dwarfs typically requires powerful, high-resolution spectroscopy with Keck or the VLT (Koester et al. 2005). The star has an infrared excess detected by WISE (Debes et al. 2011; Barber et al. 2014) at 3.4 and $4.6 \mu \mathrm{m}$, where the data are consistent with passively heated debris orbiting within the Roche limit, similar to roughly 40 other metal-enriched white dwarfs accreting from analogous discs (Farihi 2016).

Follow-up observations were obtained in 2014 April with the MMT using the Blue Channel Spectrograph. Spectra were taken through a 1 arcsec slit with the $8321 \mathrm{~mm}^{-1}$ grating in first and second order, covering 6200-8100 A at $2 \AA$ resolution and 3200$4100 \AA$ at $1 \AA$ resolution, respectively. The red spectrum consisted of four $900 \mathrm{~s}$ exposures in clear conditions, while the blue spectrum comprised three $600 \mathrm{~s}$ exposures, but intruding on twilight where significant sky signal was present. The blue data are thus of relatively modest quality, while the red spectra are superior and a combined spectrum is shown in Fig. 1. Most importantly, these modest resolution data exhibit a strong O I $7775 \AA$ absorption feature, in addition to lines of $\mathrm{Mg}$ II and $\mathrm{Si}$ II.

Additionally, medium-resolution spectra were taken in 2014 July using the double arm ISIS spectrograph on the WHT. Simultaneous blue and red spectra were taken through a 1 arcsec slit using the R1200B and R1200R gratings, with the $5300 \AA$ dichroic, resulting in two spectra covering 4500-6000 $\AA$ at a resolution of roughly $1 \AA$. The white dwarf was observed continuously for eight exposures of $900 \mathrm{~s}$ in good conditions. The ISIS spectra reveal weak Al II and $\mathrm{Si}$ II features in wavelength regions not covered by the MMT data set.

Lastly, high-resolution observations were carried out in 2015 April with the HIRESb spectrograph on Keck I. The setup was identical to that described in Zuckerman et al. (2011), covering the range 3130-5940 $\AA$. The blue cross disperser was combined with a $1.15 \operatorname{arcsec}$ slit resulting in a spectral resolving power of $R \approx$ 40000 . Reduction procedures utilized both IRAF and MAKEE. This data set reveals multiple lines of $\mathrm{Mg}$ II, $\mathrm{Al}$ II, $\mathrm{Si}$ II, $\mathrm{Ca}$ II, Ti II, Cr II, and Fe II, a portion of which is shown in Fig. 1.

All spectra were reduced in the standard fashion, by average combining each spectrum after extraction, using variance weighting for sky subtraction and rejection of bad pixels and cosmic rays.

\section{ATMOSPHERIC PARAMETERS AND ABUNDANCE PATTERNS}

The multiple spectral data sets were analysed together using white dwarf atmospheric models, where the input physics is detailed in Koester (2010). The final stellar parameters were based on spectral fits to the latest SDSS spectrum, obtained with the BOSS spectrograph. We calculated a three-dimensional model grid in $T_{\text {eff }}, \log g$, and $[\mathrm{H} / \mathrm{He}]$, keeping the latter fixed while fitting the first two parameters. This is a more stable procedure than fitting for all three parameters, since the effect of $[\mathrm{He} / \mathrm{H}]$ and $\log g$ on the spectrum is much smaller than that of the temperature. The results indicate the best fit is near $20800 \mathrm{~K}$, which was confirmed by repeating a similar fit with $\log g$ kept fixed and fitting for $T_{\text {eff }}$ and [H/He]. The final stellar parameters are given in Table 1 .

For the determination of abundances and upper limits, all available spectra (Keck, MMT, SDSS, and WHT) were used with the method of line profile fitting. Table 2 lists all the individual ions and wavelengths used for this purpose. After a good fit was approximated, models were recalculated with \pm 0.3 dex abundances, where visual inspection of each line determined the abundance and an error estimate. In the case of multiple lines, the final abundance was determined as a weighted average. Repeating the analysis with $T_{\text {eff }}$ and $\log g$ varied within the adopted errors, the systematic errors were found to be approximately $0.20 \mathrm{dex}$, but in the same direction for all elements. Relative abundance errors are 0.05 dex. The changes of the convection zone and diffusion time-scales contribute 

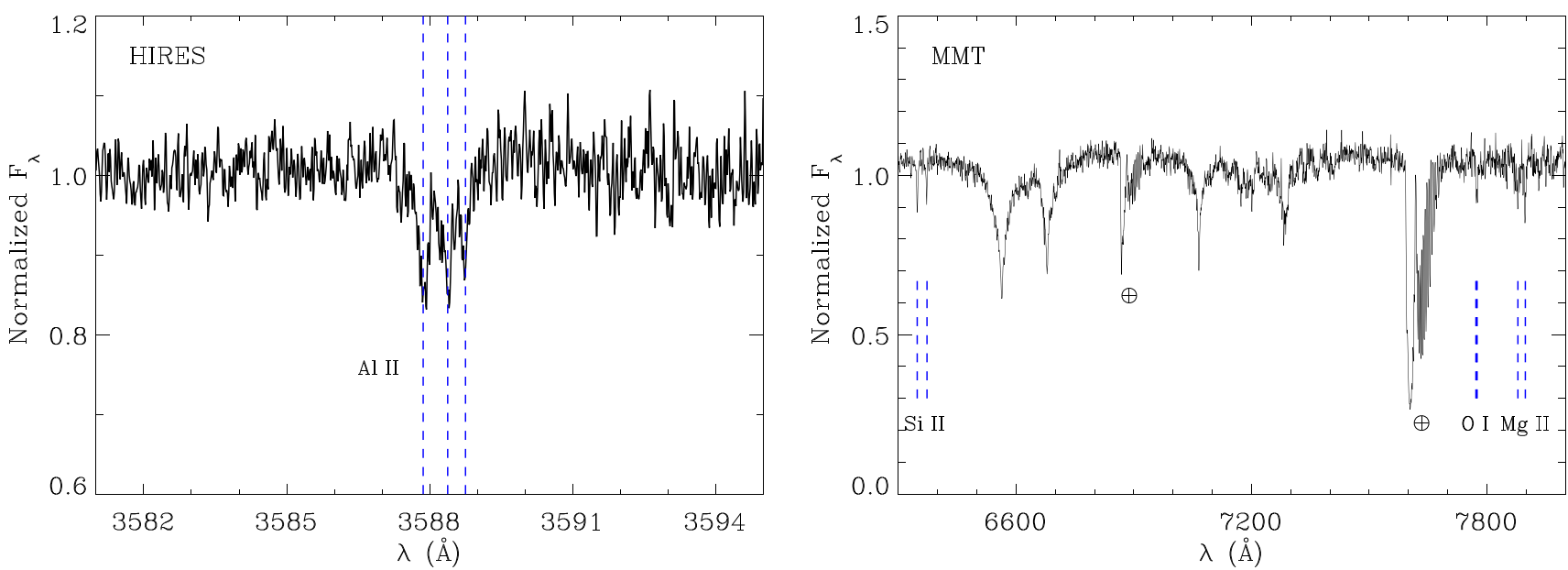

Figure 1. In the left-hand panel is a portion of the HIRES spectrum, with wavelength given in vacuum and showing a strong triplet of Al II. The right-hand panel shows a portion of the spectra obtained at the MMT, containing telluric features, $\mathrm{H} \alpha$, two He I lines, and metal lines of O I, Mg II, and Si II. Unusually, the white dwarf exhibits strong lines of both $\mathrm{H}$ and $\mathrm{He}$, indicating a mixed atmospheric composition.

Table 1. Stellar parameters for WD $1536+520$.

\begin{tabular}{lr}
\hline $\mathrm{SpT}$ & $\mathrm{DBAZ}$ \\
$g(\mathrm{AB}$ mag $)$ & 17.06 \\
$d(\mathrm{pc})$ & $217 \pm 15$ \\
$T_{\text {eff }}(\mathrm{K})$ & $20800 \pm 800$ \\
$\log g\left(\mathrm{~cm} \mathrm{~s}^{-2}\right)$ & $7.96 \pm 0.10$ \\
$\operatorname{Mass}\left(\mathrm{M}_{\odot}\right)$ & $0.58 \pm 0.05$ \\
$\log (\mathrm{H} / \mathrm{He})$ & $-1.7 \pm 0.1$ \\
$\log \left(M_{\mathrm{cvz}} / M\right)$ & -11.16 \\
Cooling age $(\mathrm{Myr})$ & $62_{-6}^{+16}$ \\
\hline
\end{tabular}

Table 2. Lines used for abundance determinations and upper limits.

\begin{tabular}{ll}
\hline Ion & Vacuum wavelength $(\AA)$ \\
\hline $\mathrm{C}_{\text {II }}$ & 4268 \\
$\mathrm{O}_{\text {I }}$ & 7775 \\
$\mathrm{Mg}_{\text {II }}$ & 4435,4482 \\
$\mathrm{Mg}_{\text {I }}$ & 5185 \\
$\mathrm{Al}_{\text {II }}$ & 3588,4664 \\
$\mathrm{Si}_{\text {II }}$ & $3854,3857,3863,4129,4132,5042,5057,6348,6373$ \\
$\mathrm{P}_{\text {II }}$ & 3509,3787 \\
$\mathrm{~S}_{\text {II }}$ & $4163,5202,5214,5455$ \\
$\mathrm{Ca}_{\text {II }}$ & $3159,3737,3934$ \\
$\mathrm{Ti}_{\text {II }}$ & $3235,3237,3239,3342,3349,3350,3362,3373,3384,3686,3762$ \\
$\mathrm{Cr}_{\text {II }}$ & $3336,3340,3343,3369,3404,3409$ \\
$\mathrm{Fe}_{\text {II }}$ & $3155,3163,3168,3178,3184,3194,3196,3211,3214,3228,3259$ \\
& $3290,3324,3469,3494,5170,5199,5200,5236,5277,5318$ \\
\hline & \\
\hline &
\end{tabular}

0.10 dex, and thus the total systematic error in relative abundances is 0.12 dex.

\subsection{Bulk composition of accreted debris}

The abundances, relative to helium, of all trace elements are given in Table 3, together with diffusion time-scales for each species. The third column compares the atmospheric, heavy element abundances in the white dwarf (relative to $\mathrm{He}$ ) in units of solar values (relative to H; Lodders 2003), demonstrating that WD 1536 nominally exceeds the solar values for nearly all detected elements. These absolute abundances surpass the previous record holder
Table 3. Abundances, masses, and accretion rates for trace elements.

\begin{tabular}{lrlrrr}
\hline Element & {$[\mathrm{Z} / \mathrm{He}]$} & $\begin{array}{l}{[\mathrm{Z} / \mathrm{He}]} \\
-\end{array}$ & $\begin{array}{r}t_{\text {diff }} \\
(\mathrm{Z} / \mathrm{H}]\end{array}$ & $\begin{array}{r}\text { Early phase } \\
X_{\mathrm{Z}} M_{\mathrm{cvz}} \\
\left(10^{19} \mathrm{~g}\right)\end{array}$ & $\begin{array}{r}\text { Steady state } \\
\left(10^{9} \mathrm{~g} \mathrm{~s}^{-1}\right)\end{array}$ \\
\hline $\mathrm{H}$ & -1.70 & & $\infty$ & 4.012 & \\
$\mathrm{C}$ & $\leq-4.2$ & & 261 & $\leq 0.152$ & $\leq 0.183$ \\
$\mathrm{O}$ & -3.40 & -0.09 & 302 & 1.276 & 1.329 \\
$\mathrm{Mg}$ & -4.06 & +0.39 & 166 & 0.424 & 0.804 \\
$\mathrm{Al}$ & -5.38 & +0.16 & 141 & 0.023 & 0.050 \\
$\mathrm{Si}$ & -4.32 & +0.14 & 122 & 0.269 & 0.692 \\
$\mathrm{P}$ & $\leq-7.1$ & & 120 & $\leq 0.001$ & $\leq 0.001$ \\
$\mathrm{~S}$ & $\leq-5.4$ & & 126 & $\leq 0.026$ & $\leq 0.064$ \\
$\mathrm{Ca}$ & -5.28 & +0.38 & 146 & 0.042 & 0.091 \\
$\mathrm{Ti}$ & -6.84 & +0.24 & 126 & 0.001 & 0.003 \\
$\mathrm{Cr}$ & -5.93 & +0.43 & 103 & 0.012 & 0.037 \\
$\mathrm{Fe}$ & -4.50 & +0.03 & 97 & 0.354 & 1.148 \\
$\Sigma$ & & & & 2.40 & 4.16 \\
\hline
\end{tabular}

Notes. Errors in abundance determinations are typically $0.1-0.2$ dex. The fifth column is the mass of each element residing in the stellar convection zone, which consists of $8.0 \times 10^{21} \mathrm{~g}$ of helium and $4.0 \times 10^{19} \mathrm{~g}$ of hydrogen. Due to their continual sinking, the mass of heavy elements within the convection zone represents a minimum mass for the parent body. The metal-to-metal ratios within the planetary debris for the early-phase and steady-state regimes are derived directly from the values in the fifth and sixth columns, respectively. The diffusion time-scales are a sensitive function of $T_{\text {eff }}$ within the range of acceptable temperatures for WD 1536, and thus contribute some additional uncertainty to the derived abundance ratios.

SDSS J073842.56+183509.6 (Dufour et al. 2010) by a factor of 3-10, and GD 362 by over an order of magnitude (Xu et al. 2013).

Also calculated are the mass of each element present in the photosphere of the star, which is equivalent to the mass fraction of a given element $X_{\mathrm{Z}}$, multiplied by the mass of the convection zone $M_{\mathrm{cvz}}$. If WD 1536 is in an early phase of accretion, where less than a single diffusion time-scale has expired since the onset of atmospheric pollution, then the metal-to-metal abundances of the infalling debris are exactly mirrored by those in the atmosphere and given in the fifth column. If instead pollution has been ongoing for at least five diffusion time-scales (Koester 2009), then the system is in a steady-state balance between accretion and diffusion and the abundance ratios are reflected in the sixth column. For all detected 


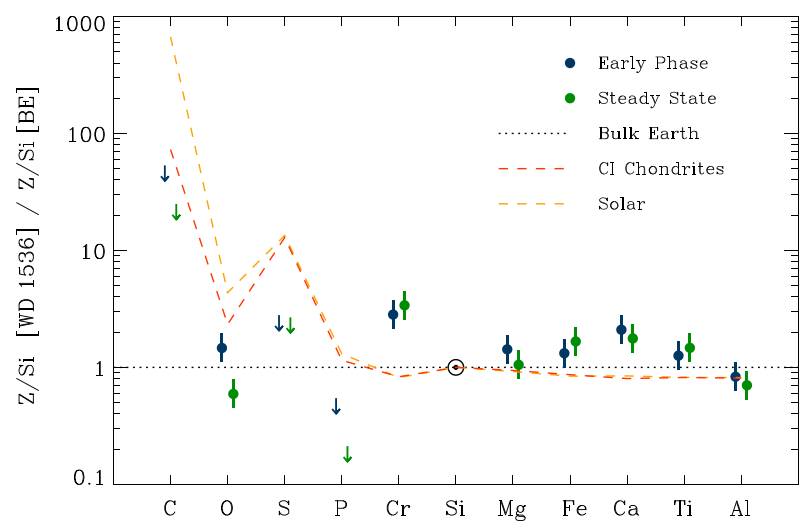

Figure 2. Derived number abundances for the planetary debris polluting the outer layers of WD 1536. Shown in blue and green, respectively, are the early-phase and steady-state abundances of each heavy element relative to $\mathrm{Si}$, divided by the same ratio as determined for the bulk Earth (Allègre et al. 2001). A typical uncertainty in the derived ratios is 0.12 dex, and error bars of this size are overplotted. Also plotted are the same ratios for CI chondrites and the Sun (Lodders 2003), demonstrating that these compositions can be confidently ruled out for WD 1536 based on ground-based data alone. At face value, the disrupted parent body appears broadly similar to the bulk Earth, with notably high chromium.

elements but oxygen, the metal-to-metal ratios show little variation between the early phase and steady-state solutions.

In Fig. 2 are plotted both the early phase and steady-state abundances of heavy elements, relative to silicon and normalized to the bulk Earth values from Allègre, Manhès \& Lewin (2001). As can be seen, the debris orbiting and polluting WD 1536 is bulk Earth-like in the major rock-forming elements to within a factor of around 2. There is a notable enhancement in chromium, yet an apparent deficit in phosphorous. This two-fold deviation in opposite directions is difficult to reconcile, as both chromium and phosphorous are siderophiles with similar condensation temperatures (Lodders 2003). Similar enhancements in chromium have been seen in the white dwarfs PG 0843+516 and GALEX J193156.8+011745 (Gänsicke et al. 2012) - together with bulk Earth or higher phosphorous abundances, as expected - but are otherwise not commonly seen in polluted white dwarfs (Jura et al. 2012; Xu et al. 2014). Because phosphorous has only been detected in white dwarfs at ultraviolet wavelengths, the upper limit derived for WD 1536 from optical data may be uncertain. With this caveat, the data are consistent with the accretion of substantial core-like material.

\subsection{Oxygen excess and hydrogen accreted from $\mathrm{H}_{2} \mathrm{O}$}

The total oxygen budget can be evaluated by accounting for all the expected oxides originating in planetary solids (Klein et al. 2010; Farihi et al. 2013a). In the early-phase and steady-state scenarios, oxygen is first assumed to be carried exclusively by $\mathrm{MgO}, \mathrm{Al}_{2} \mathrm{O}_{3}$, $\mathrm{SiO}_{2}, \mathrm{CaO}$, and $\mathrm{FeO}$ within the debris. There are three possible outcomes from such an analysis.

(i) Insufficient oxygen to account for metal oxides. This outcome can imply that iron was delivered not as $\mathrm{FeO}$ but substantially as metal.

(ii) An oxygen budget as expected for oxides in planetary solids. In this case, the debris is rocky and poor both in water ices and hydrated minerals resulting from aqueous alteration.

(iii) Excess oxygen beyond that of anhydrous minerals alone. In this case, $\mathrm{H}_{2} \mathrm{O}$ is the most likely source of the oxygen surplus.
Table 4. Oxide, iron metal, and water mass fractions.

\begin{tabular}{lrr}
\hline Oxygen carrier & Early phase & Steady state \\
\hline $\mathrm{MgO}$ & 0.22 & 0.40 \\
$\mathrm{Al}_{2} \mathrm{O}_{3}$ & 0.02 & 0.03 \\
$\mathrm{SiO}_{2}$ & 0.24 & 0.59 \\
$\mathrm{CaO}$ & 0.01 & 0.03 \\
$\mathrm{FeO}^{a}$ & 0.08 & 0.00 \\
$\mathrm{O}^{a}$ excess & 0.43 & - \\
$\mathrm{H}_{2} \mathrm{O}$ in debris & 0.25 & - \\
$\mathrm{Fe}$ in metal & 0.00 & 1.00
\end{tabular}

Notes. ${ }^{a}$ Upper limit for $\mathrm{FeO}$.

The first five rows assume oxygen is carried to maximum capacity by all the major rock-forming elements, but in fact iron can also be in pure metal or iron-nickel alloy with no oxygen. The nominal oxygen budget in the steady state is unphysical unless 100 per cent of the total iron is carried as metal, and the nominal $\mathrm{O} / \mathrm{Si}$ and $\mathrm{O} / \mathrm{Mg}$ ratios are marginally higher than tabulated.

Carbon can confidently be ignored as an oxygen carrier for the following reasons. First, carbon has been found to be significantly depleted relative to solar and volatile-rich, cometary abundance patterns in nearly all polluted white dwarfs where measurements are available (Jura 2006; Gänsicke et al. 2012; Jura et al. 2012; Farihi, Gänsicke \& Koester 2013b; Koester, Gänsicke \& Farihi 2014; Wilson et al. 2016). Secondly, $\mathrm{CO}$ and $\mathrm{CO}_{2}$ are no more than 5-10 per cent of the volatile content of solar system comets, which are dominated by water ice (Binzel, Hanner \& Steel 2000). Thirdly, for WD 1536 in particular, the upper limit carbon abundance suggests that it cannot be a significant source of excess oxygen.

Table 4 evaluates the nominal oxygen budget of WD 1536 for both an early-phase and steady-state accretion history. In the steady state, there is insufficient oxygen to account for $\mathrm{Mg}, \mathrm{Al}, \mathrm{Si}, \mathrm{Ca}$, and Fe bound in oxides - only if 100 per cent of the iron was delivered as metal or alloy can the oxygen budget be considered balanced and physical. In this case, the nominal oxygen abundance still requires a modest, 5-10 per cent increase to account for the other elements ( $\mathrm{Mg}, \mathrm{Al}, \mathrm{Si}, \mathrm{Ca}$ ) that only form rocks, but such leeway is well within the uncertainties. This is another strong indication that the material orbiting and polluting the white dwarf has a substantial core-like component. Of the total iron mass present in the Earth, metallic Fe in the core is thought to represent 87 per cent, whereas $\mathrm{Fe}$ in the mantle and crust is only 13 per cent (McDonough 2000), some of which is also metal. Thus, the scenario where the iron in WD 1536 was contained essentially in pure metals or alloys is plausible. Within the derived photospheric abundance errors, a steady-state solution without any iron oxides would readily allow for solutions where the parent body contained water ice or hydrated minerals.

While the range of allowed abundance ratios also permits solutions without any excess oxygen, the striking hydrogen abundance in WD 1536 must be considered, and which clearly favours a waterrich interpretation. While an early phase of accretion predicts an oxygen excess and thus the need for $\mathrm{H}_{2} \mathrm{O}$ within the planetary debris, the heavy element settling times are relatively short, and thus catching the star in this phase is less likely. If discs last for at least $10^{5} \mathrm{yr}$ (Girven et al. 2012), then the probability that WD 1536 is not yet in a steady-state phase of accretion is less than 1 per cent. The total hydrogen mass within the stellar atmosphere is $4.0 \times 10^{19} \mathrm{~g}$, and could have been delivered by an asteroid with total mass a few to several times $10^{21} \mathrm{~g}$ and which was 5-10 per cent $\mathrm{H}_{2} \mathrm{O}$ by mass. 


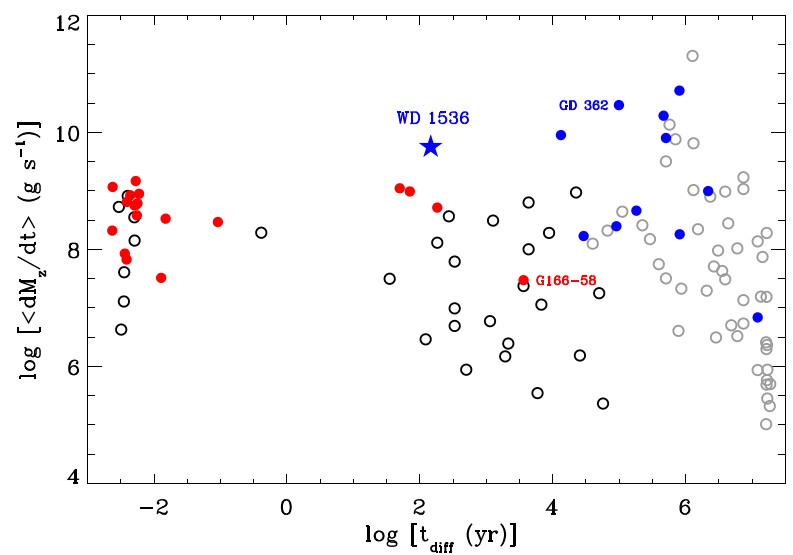

Figure 3. Accretion rate versus diffusion time-scale for WD 1536 and a large sample of metal-enriched white dwarfs observed with Spitzer (Bergfors et al. 2014). For consistency, all plotted rates and time-scales are based solely on Ca, following the method outlined in Farihi et al. (2012) with updated diffusion data (Koester 2009). The hydrogen-rich stars are shown as red filled and black open circles, while the helium-rich stars are shown as blue filled and grey open circles; filled symbols correspond to the detection of infrared excess. Within each atmospheric class, left to right represents decreasing $T_{\text {eff. }}$. Remarkably, WD 1536 sits in a region that is otherwise exclusively occupied by stars with hydrogen atmospheres. G166-58 is the coolest white dwarf with a hydrogen-rich atmosphere and an infrared excess.

This would be consistent with the lower mass limit of $4.2 \times 10^{19} \mathrm{~g}$ from the heavy elements alone.

While uncertain, the totality of data discussed in this section favours the deposition of $\mathrm{H}_{2} \mathrm{O}$ on to the stellar surface and is carried by the parent body whose debris now orbits the star. In the next section, the anomalously high trace hydrogen abundance is shown to be transient, thus strengthening this interpretation.

\subsection{Anomalous diffusion time-scales and trace $\mathbf{H}$}

The mass of the convection zone in WD 1536 is tiny $-10^{6}$ times smaller than those within the bulk of known polluted white dwarfs with helium atmospheres. There are two reasons for this. First, the $T_{\text {eff }}$ and $60 \mathrm{Myr}$ cooling age means the star is experiencing the early stages of convection zone growth (Paquette et al. 1986). In fact, with $T_{\text {eff }}>20000 \mathrm{~K}$ this star is the warmest and the youngest helium-rich white dwarf to show metals due to ongoing accretion. Secondly, the anomalously high fraction of hydrogen leads to a significant reduction in the depth of the outer layers relative to a pure helium composition, by a factor of approximately 30 .

These facts conspire to make the stellar atmosphere physically similar to a typical $10000 \mathrm{~K}$ hydrogen-rich white dwarf, with comparable diffusion time-scales. Fig. 3 plots WD 1536 together with a sample of polluted white dwarfs observed with Spitzer, as a function of their inferred accretion rates and sinking time-scales based on Ca II detections (Bergfors et al. 2014). WD 1536 lies above three hydrogen-rich stars whose discs have been detected in the infrared (von Hippel et al. 2007; Farihi et al. 2010b), and has an even shorter sinking time-scale than G166-58 (Farihi, Zuckerman \& Becklin 2008). This so-far unique position for a helium-dominated star strongly suggests (1) it is accreting at a high rate in a steady state, and (2) that the older, cooler stars with similar sinking timescales do not often experience similarly high rates of accretion. If this interpretation is correct, it would support a decreasing trend of planetary dynamical activity in the post-main sequence, as measured
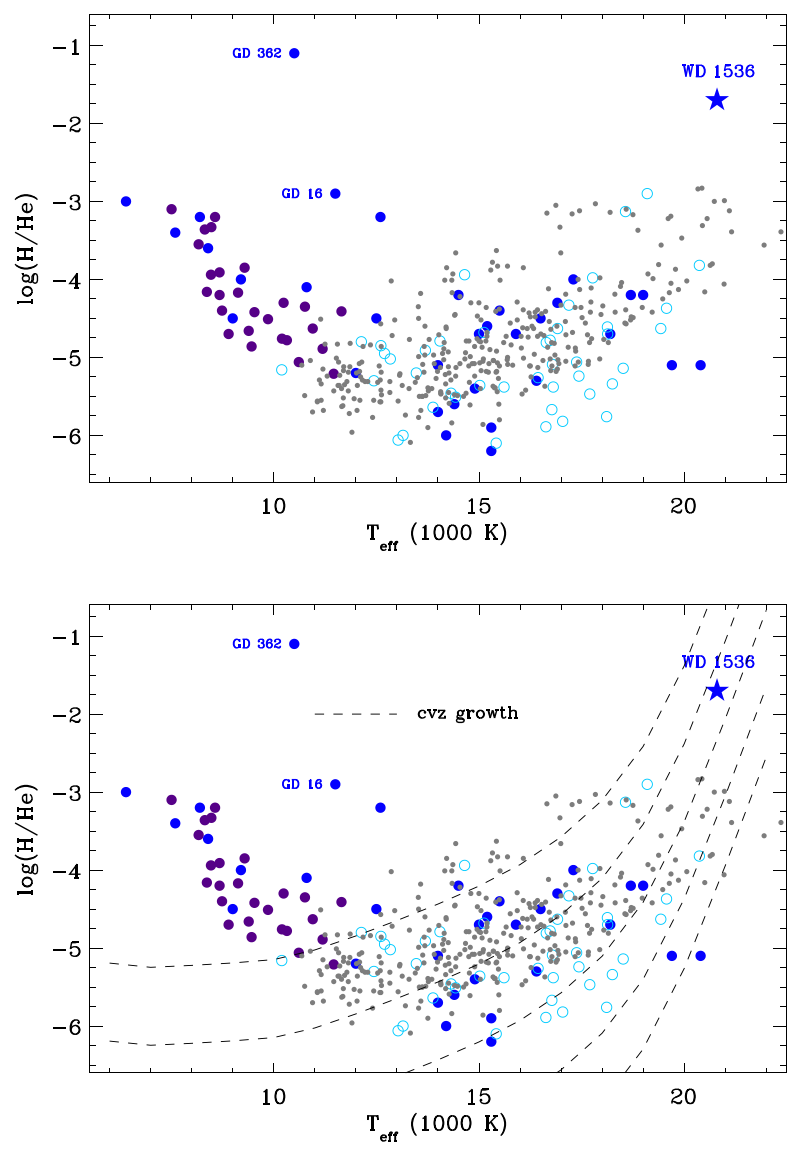

Figure 4. Relatively cool, helium atmosphere white dwarfs with detected hydrogen absorption. Light blue open circles are nearby stars from Bergeron et al. (2011) and Voss et al. (2007), while the grey dots are a more distant and abundant sample from Koester \& Kepler (2015). Filled purple circles are stars with strong $\mathrm{Ca}$ II from Dufour et al. (2007), and filled dark blue circles are Fig. 3 stars with matching criteria. The upper panel demonstrates the anomalous nature of WD 1536, whose $\mathrm{H} / \mathrm{He}$ ratio is over an order of magnitude higher than any comparable object and second only to GD 362 (Zuckerman et al. 2007). Overplotted in the lower panel are tracks demonstrating the directly proportional change in $\mathrm{H} / \mathrm{He}$ due to the growth of the convection zone over time in pure helium atmosphere stars (Koester 2009), shown for five orders of magnitude of initial $[\mathrm{H} / \mathrm{He}]=1.0,0,-1.0,-2.0$, -3.0 . These calculations assume complete mixing of the outer layers.

by the mass influx of planetesimals towards the white dwarf host, consistent with theoretical predictions (Veras et al. 2013; Mustill, Veras \& Villaver 2014)

Fig. 4 highlights the exceptional H/He in WD 1536. The upper panel plots samples of helium-rich white dwarfs with trace hydrogen, detected directly through Balmer absorption features (typically only $\mathrm{H} \alpha) .{ }^{1}$ Interestingly, a substantial fraction of the plotted stars is also polluted with heavy elements, although a strong bias is present at $T_{\text {eff }} \lesssim 12000 \mathrm{~K}$. In this cooler temperature range, He I absorption rapidly becomes too weak to detect in low- and medium-resolution spectra, whereas strong $\mathrm{Ca}$ II absorption can indicate a helium-rich atmosphere (Dufour et al. 2007). At the warmer end of the temperatures shown in Fig. 4, the bias towards metal detection is not an issue. Caution should be used when viewing Fig. 4; the plotted stars do not represent an evolutionary sequence, and selection biases play

\footnotetext{
${ }^{1}$ WD 1536 displays strong Balmer lines up to and including $\mathrm{H} \delta$.
} 
a large role. That being said, the cooler stars with substantial hydrogen are either born with substantially more massive reservoirs than can currently be inferred in earlier evolutionary stages, or accrete H-rich planetary material (Farihi et al. 2010a).

\section{$3.4 \mathrm{H} / \mathrm{He}$ evolution}

Assuming complete mixing of the outer stellar layers, the lower panel of Fig. 4 plots tracks of constant hydrogen mass within otherwise-pure helium atmosphere white dwarfs, as a function of temperature (Koester 2009). In this simple model, where no stratification occurs between hydrogen and helium, the observational signature of most fixed masses of hydrogen at $T_{\text {eff }} \approx 20000 \mathrm{~K}$ will gradually disappear from white dwarfs with helium-dominated atmospheres. The fact that some stars retain (or regain) substantial hydrogen masses at later times, is a well-known problem in white dwarf atmospheric evolution (MacDonald \& Vennes 1991). While this general topic is beyond the scope of this paper, two distinct possibilities are (1) hydrogen is accreted over long time-scales, or (2) primordial hydrogen floats over a deeper helium reservoir, and is later mixed into the photosphere. In the latter scenario, stars will appear hydrogen rich at sufficiently warm temperatures and later reveal themselves to be helium dominated (Fontaine \& Wesemael 1987; Bergeron et al. 2011). Currently, there is more observational support for the primordial model, with at least 3/4 of helium atmosphere white dwarfs showing traces of hydrogen (Koester \& Kepler 2015).

Thus, in the absence of continued accretion, WD 1536 will have both its metals and trace hydrogen wiped clean from its photosphere. Without the influence of ongoing, external pollution, the heavy elements will completely sink beneath the photosphere within a few $10^{3} \mathrm{yr}$ at most. But over longer time-scales, the remarkably high abundance of atmospheric hydrogen will be drowned by the deepening helium convection zone. By the time WD 1536 has cooled to $15000 \mathrm{~K}$ in $140 \mathrm{Myr}$, the mass of the convection zone will have grown by four orders of magnitude and exhibit a trace hydrogen abundance $\log (\mathrm{H} / \mathrm{He})=-5.7$. At this stage, the star will either appear as a fairly average helium-rich white dwarf, where hydrogen is difficult or impossible to detect in modest resolution spectra due to its apparent faintness relative to the nearby samples shown in Fig. 4. When the star has cooled to $12000 \mathrm{~K}$ after another $190 \mathrm{Myr}$, it will certainly not have detectable hydrogen. The unavoidable conclusion is that this white dwarf is being witnessed at a special time, in a transient phase, and the hydrogen is related to the orbiting planetary debris, and thus water is likely present.

Considering that WD 1536 may have only accreted $\sim 10^{19} \mathrm{~g}$ of hydrogen on to its atmosphere and $\log (\mathrm{H} / \mathrm{He})=-1.7$, it can be seen that if a significantly larger and water-rich parent body had been deposited, then hydrogen could (temporarily) have become the dominant atmospheric constituent. For example, the disrupted asteroids polluting GD 61 or SDSS 1242 might have delivered $\sim 10^{21}$ or $\sim 10^{23} \mathrm{~g}$ of hydrogen, respectively, resulting in abundances of $\log (\mathrm{H} / \mathrm{He}) \sim 0$ and $\log (\mathrm{H} / \mathrm{He}) \sim+2$. In both cases, WD 1536 would temporarily appear as a hydrogen-rich star despite being dominated by the underlying helium. Therefore, the accretion of water-rich planetary debris has the potential to have an observable effect on $\mathrm{H} / \mathrm{He}$ white dwarf spectral evolution.

\section{CONCLUSION}

The young, helium-atmosphere, white dwarf WD 1536 exhibits the highest abundances of heavy elements yet seen among polluted hosts of evolved planetary systems. In addition to the broadly solar abundances of the major rock-forming elements, $\mathrm{O}, \mathrm{Mg}, \mathrm{Al}, \mathrm{Si}, \mathrm{Ca}$, and $\mathrm{Fe}$, this star also has a remarkably high trace hydrogen abundance of $\log (\mathrm{H} / \mathrm{He})=-1.7$. Considering the (1) abundance pattern of heavy elements, (2) the anomalously high trace hydrogen, and (3) the transient detectability of both the metals and the hydrogen, the most realistic conclusion is that the parent body whose debris is both orbiting and polluting WD 1536 contained traces of $\mathrm{H}_{2} \mathrm{O}$.

The thinness of the convection zone is a result of relative youth and relatively high mass of trace hydrogen within a heliumdominated atmosphere. Due to these combined facts, the outer layers of WD 1536 essentially behave as a hydrogen-rich white dwarf, with metal-sinking time-scales of only a few hundred years at most, hence supporting a steady-state interpretation of the metal abundances. If these are indeed in a steady state, then WD 1536 has the highest instantaneous accretion rate yet observed among polluted white dwarfs.

\section{ACKNOWLEDGEMENTS}

JF thanks S. Desch for feedback on a draft manuscript. The authors acknowledge both the MMT and WHT (Service programme SW2014a39) for the expedient use of their Directors' time, without which these results would not have been possible, and an anonymous reviewer for feedback that improved the quality of the manuscript. JF gratefully acknowledges the support of the STFC via an Ernest Rutherford Fellowship. This research was supported in part by a NASA grant to UCLA, and by an NSF pre-doctoral fellowship to LV. The research leading to these results has received funding from the ERC under the European Union's 7th Framework Programme no. 320964 (WDTracer).

\section{REFERENCES}

Allègre C., Manhès G., Lewin É., 2001, Earth Planet. Sci. Lett., 185, 49 Balayan S. K., 1997, Astrophysics, 40, 211

Barber S. D., Kilic M., Brown W. R., Gianninas A., 2014, ApJ, 786, 77

Bergeron P. et al., 2011, ApJ, 737, 28

Bergfors C., Farihi J., Dufour P., Rocchetto M., 2014, MNRAS, 444, 2147

Binzel R. P., Hanner M. S., Steel D. I., 2000, in Cox A. N., ed., Allen's Astrophysical Quantities, 4th edn. Am. Inst. Phys., New York, p. 315

Bonsor A., Mustill A. J., Wyatt M. C., 2011, MNRAS, 414, 930

Debes J. H., Hoard D. W., Wachter S., Leisawitz D. T., Cohen M., 2011, ApJS, 197, 38

Debes J. H., Walsh K., Stark C., 2012, ApJ, 747, 148

Dufour P. et al., 2007, ApJ, 663, 1291

Dufour P., Kilic M., Fontaine G., Bergeron P., Lachapelle F. R., Kleinman S. J., Leggett S. K., 2010, ApJ, 719, 803

Eisenstein D. J. et al., 2006, ApJS, 167, 40

Farihi J., 2016, New Astron. Rev., 71, 9

Farihi J., Zuckerman B., Becklin E. E., 2008, ApJ, 674, 431

Farihi J., Jura M., Zuckerman B., 2009, ApJ, 694, 805

Farihi J., Barstow M. A., Redfield S., Dufour P., Hambly N. C., 2010a, MNRAS, 404, 2123

Farihi J., Jura M., Lee J. E., Zuckerman B., 2010b, ApJ, 714, 1386

Farihi J., Gänsicke B. T., Wyatt M. C., Girven J., Pringle J. E., King A. R., 2012, MNRAS, 424, 464

Farihi J., Gänsicke B. T., Koester D., 2013a, Science, 342, 218

Farihi J., Gänsicke B. T., Koester D., 2013b, MNRAS, 432, 1955

Fontaine G., Wesemael F., 1987, in Philip A. G. D., Hayes D. S., Liebert J., eds, IAU Colloq. 95: 2nd Conference on Faint Blue Stars, Davis Press, Schenectady, p. 319

Frewen S. F. N., Hansen B. M. S., 2014, MNRAS, 439, 2442 
Gänsicke B. T., Marsh T. R., Southworth J., Rebassa-Mansergas A., 2006, Science, 314, 1908

Gänsicke B. T., Koester D., Farihi J., Girven J., Parsons S. G., Breedt E., 2012, MNRAS, 424, 333

Girven J., Gänsicke B. T., Steeghs D., Koester D., 2011, MNRAS, 417, 1210

Girven J., Brinkworth C. S., Farihi J., Gänsicke B. T., Hoard D. W., Marsh T. R., Koester D., 2012, ApJ, 749, 154

Jura M., 2006, ApJ, 653, 613

Jura M., Xu S., 2012, AJ, 143, 6

Jura M., Young E. D., 2014, Annu. Rev. Earth Planet. Sci., 42, 45

Jura M., Farihi J., Zuckerman B., 2007, ApJ, 663, 1285

Jura M., Xu S., Klein B., Koester D., Zuckerman B., 2012, ApJ, 750, 69

Klein B., Jura M., Koester D., Zuckerman B., Melis C., 2010, ApJ, 709, 950

Koester D., 2009, A\&A, 498, 517

Koester D., 2010, Mem. Soc. Astron. Ital., 81, 921

Koester D., Kepler S. O., 2015, A\&A, 583, A86

Koester D., Rollenhagen K., Napiwotzki R., Voss B., Christlieb N., Homeier D., Reimers D., 2005, A\&A, 432, 1025

Koester D., Gänsicke B. T., Farihi J., 2014, A\&A, 566, A34

Lodders K., 2003, ApJ, 591, 1220

McDonough W. F., 2000, in Teisseyre R., Majewski E., eds, Earthquake Thermodynamics and Phase Transformation in the Earth's Interior. Academic Press, San Diego, p. 5

MacDonald J., Vennes S., 1991, ApJ, 371, 719

Morbidelli A., Chambers J., Lunine J. I., Petit J. M., Robert F., Valsecchi G. B., Cyr K. E., 2000, Meteorit. Planet. Sci., 35, 1309

Mustill A. J., Veras D., Villaver E., 2014, MNRAS, 437, 1404

Paquette C., Pelletier C., Fontaine G., Michaud G., 1986, ApJS, 61, 197
Raddi R., Gänsicke B. T., Koester D., Farihi J., Hermes J. J., Scaringi S., Breedt E., Girven J., 2015, MNRAS, 450, 2083

Rocchetto M., Farihi J., Gänsicke B. T., Bergfors C., 2015, MNRAS, 449, 574

Stepanian J. A., Chavushyan V. H., Carrasco L., Tovmassian H. M., Erastova L. K., 1999, PASP, 111, 1099

van Maanen A., 1917, PASP, 29, 258

Vanderburg A. et al., 2015, Nature, 526, 546

Veras D., Mustill A., Bonsor A., Wyatt M. C., 2013, MNRAS, 431, 1686

Veras D., Leinhardt Z. M., Eggl S., Gänsicke B. T., 2015, MNRAS, 451, 3453

von Hippel T., Kuchner M. J., Kilic M., Mullally F., Reach W. T., 2007, ApJ, 662, 544

Voss B., Koester D., Napiwotzki R., Christlieb N., Reimers D., 2007, A\&A, 470, 1079

Wilson D. J., Gänsicke B. T., Farihi J., Koester D., 2016, MNRAS, 459, 3282

Xu S., Jura M., Klein B., Koester D., Zuckerman B., 2013, ApJ, 766, 132

Xu S., Jura M., Koester D., Klein B., Zuckerman B., 2014, ApJ, 783, 79

Zuckerman B., 2015, in Dufour P., Bergeron P., Fontaine G., eds, ASP Conf. Ser. Vol. 493, 19th European Workshop on White Dwarfs. Astron. Soc. Pac., San Francisco, p. 291

Zuckerman B., Koester D., Melis C., Hansen B. M. S., Jura M., 2007, ApJ, 671,872

Zuckerman B., Koester D., Dufour P., Melis C., Klein B., Jura M., 2011, ApJ, 739, 101

This paper has been typeset from a $\mathrm{T}_{\mathrm{E}} \mathrm{X} / \mathrm{L} \mathrm{T} \mathrm{E} \mathrm{X}$ file prepared by the author. 